\title{
Mehr Herzinfarkte unter Clarithromycin
}

\author{
In den ersten zwei Wochen der Einnahme von Clarithromycin sind das Risiko für kardio- \\ vaskuläre Komplikationen und die Sterblichkeit um das 1,5- bis 3,5-Fache erhöht.
}

_ In einer Studie wurde überprüft, ob das Makrolidantibiotikum Clarithromycin kardiovaskuläre Komplikationen begünstigt. 108.988 Patienten mit Clarithromycin-Therapie wurden mit 217.793 Patienten verglichen, die nach Alter, Geschlecht und Einnahmezeitpunkt übereinstimmten, aber Amoxicillin eingenommen hatten. Primärer Endpunkt

\section{Tab. 1 Relative Risiken unter Clarithromycin im Vergleich zu Amoxicillin über einen Beobach- tungszeitraum von drei Jahren}

\begin{tabular}{|l|l|l|}
\hline Tag & $\mathbf{1 - 1 4}$ & ab 15 \\
\hline Herzinfarkte & 3,66 & 1,05 \\
\hline Arrhythmien & 2,22 & 1,03 \\
\hline Schlaganfälle & 1,11 & 1,15 \\
\hline Gesamtmortalität & 1,97 & 0,96 \\
\hline Kardiale Mortalität & 1,67 & 0,98 \\
\hline Nicht kardiale Mortalität & 2,10 & 1,02 \\
\hline
\end{tabular}

waren Herzinfarkte, außerdem wurden Daten zu Herzrhythmusstörungen und Schlaganfällen sowie zur Mortalität, aufgeschlüsselt nach kardialen und nicht kardialen Ursachen, erhoben.

Alle Endpunkte mit Ausnahme der Schlaganfälle waren nach der Berücksichtigung möglicher Einflussfaktoren in den ersten 14 Tagen nach Beginn der Einnahme von Clarithromycin um das 1,67- bis 3,66-Fache jeweils signifikant erhöht. Besonders gefährdet waren Patienten jenseits des 75 . Lebensjahres, Hypertoniker und Diabetiker. Das Risiko bestand nur während der Einnahme des Antibiotikums in den ersten 14 Tagen, während langfristig kein Unterschied zu Amoxicillin bestand. Betrachtet wurden die Zeiträume 15-30, 31-90 und 91-365 Tage sowie die Folgezeit bis zu drei Jahren. Die Indikation für die Verordnung war unerheblich.

\footnotetext{
- Wong AY, Root A, Douglas IJ et al. Cardiovascular outcomes associated with use of clarithromycin: population based study. BMJ. 2016;352:h6926
}

\section{KOMMENTAR}

Clarithromycin wird häufig bei Atemwegsinfektionen und zur Eradikation von Helicobacter pylori verordnet. Zum kardiovaskulären Risiko dieses Antibiotikums gibt es widersprüchliche Hinweise. Immerhin nennt die Fachinformation als Anwendungsbeschränkung "koronare Gefäßerkrankungen". Im Vergleich zu Amoxicillin war in der vorliegenden Kohortenstudie das Risiko für kardiovaskuläre Erkrankungen (ausgenommen Schlaganfälle) sowie die Mortalität in den ersten 14 Tagen, also in der Regel während der Einnahme, signifikant erhöht.

Aus den Zahlen kann man errechnen, dass in diesem Zeitraum 1-2 von 100 Patienten eine der genannten Komplikationen erleiden. $\mathrm{Pa}$ tienten, die über 75 Jahre alt sind und kardiovaskuläre Risiken aufweisen, sind stärker gefährdet. Ein Langzeitrisiko kann ausgeschlossen werden. Bei der Verordnung von Clarithromycin sollten also das individuelle Risiko, das NutzenRisikoVerhältnis und mögliche Alternativen berücksichtigt werden.

Prof. Dr. med. H. Holzgreve

\section{Rupturierte Baker-Zyste}

Ein 62-jähriger Mann stellte sich wegen plötzlich und spontan aufgetretener, starker Schmerzen in der rechten Wade vor. Bei der körperlichen Untersuchung imponierte an dieser Stelle eine ausgeprägte Schwellung und Rötung. Die klinische Verdachtsdiagnose einer rupturierten Baker-Zyste konnte mittels Ultraschall bestätigt werden. Eine tiefe Beinvenenthrombose wurde ausgeschlossen. Unter sonografischer Kontrolle wurde der Zysteninhalt weitgehend abpunktiert.

Baker-Zysten entstehen meistens zwischen dem medialen Kopf des M. gastrocnemius und des M. semimembranosus. Bei einer Ruptur breitet sich der Zysteninhalt in der Muskelloge aus und verursacht eine Schwellung medial am Unterschenkel. Zur Schmerzerleichterung stehen die Patienten typischerweise mit leicht gebeugten Knie und gestrecktem Sprunggelenk, um den M. gastrocnemius zu entlasten. So tat es auch der Patient im vorliegenden Fall (Abb.). Der Zysteninhalt ruft häufig eine entzündliche Reaktion im umgebenden Gewebe hervor. Die Therapie ist in der Regel konservativ und besteht in Hochlagerung des Beins, lokaler Wärmebehandlung und der Gabe von nicht-steroidalen Antirheumatika.

Prof. Dr. med. H. S. FüeßI

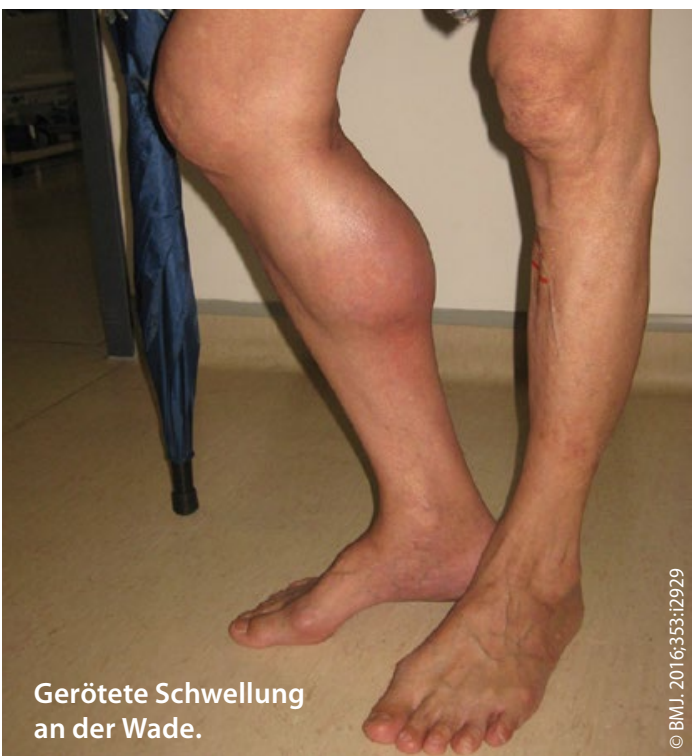

